\title{
Kunststoffe
} in der Medizintechnik 
https://doi.org/10.51202/9783182443469-1

Generiert durch IP '172.22.53.54', am 26.04.2023, 15:00:42 
KUNSTSTOFFTECHNIK

\section{Kunststoffe in der Medizinfechnik}

Herausgeber: VDI Wissensforum GmbH 
Bibliographische Information der Deutschen Bibliothek

Die Deutsche Bibliothek verzeichnet diese Publikation in der Deutschen Nationalbibliographie; detaillierte bibliographische Daten sind im Internet unter http://dnb. ddb. de abrufbar.

\section{Bibliographic information published by the Deutsche Bibliothek}

(German National Library)

The Deutsche Bibliothek lists this publication in the Deutsche Nationalbibliographie

(German National Bibliography); detailed bibliographic data is available via Internet at http:// dnb.ddb.de.

\section{(C) VDI Verlag GmbH . Düsseldorf 2016}

Alle Rechte, auch das des auszugweisen Nachdruckes, der auszugweisen oder vollständigen photomechanischen Wiedergabe (Photokopie, Mikrokopie) und das der Übersetzung, vorbehalten.

Printed in Germany

ISBN 978-3-18-234346-2 
VDI-Programmausschuss Kunststoffe in der Medizintechnik

Dipl.-Ing. (FH) Daniel Behrens, Clariant Masterbatches, Darmstadt Christian Boos, Waldorf Technik GmbH \& Co. KG, Engen

Herbert Busslinger, RIWISAAG, Hägglingen, Schweiz

Dr. Thomas Feldmann, DQS Medizinprodukte $\mathrm{GmbH}$, Frankfurt am Main

Dipl.-Ing. Thomas Hörl MBA, KraussMaffei Technologies $\mathrm{GmbH}$, München

Dipl.-Ing. Martin Itrich, 3M Deutschland $\mathrm{GmbH}$, Wuppertal (Vorsitz)

Dipl.-Ing. (FH) Gerold Keller, KEBO AG, Neuhausen, Schweiz

Prof. Dr. Günter Lorenz, Hochschule Reutlingen

Prof. Dr.-Ing. Daniel Paßmann, FH Bielefeld University of Applied Sciences

Dr.-Ing. Gösta Pretel, Hoefer \& Sohn $\mathrm{GmbH}$, Fürth

Dr. Stefan Roth, B. Braun Melsungen AG, Melsungen

Dr.-Ing. Jörn-Eric Schulz, Balda Medical GmbH, Bad Oeynhausen

Prof. Dr. Ing. Thomas Seul, Hochschule Schmalkalden

André Stutz, Wild \& Küpfer AG, Schmerikon, Schweiz

Thomas Wiles, BASF SE, Ludwigshafen

Dipl.-Inf. Gerhard Würth, ARBURG GmbH + Co KG, Loßburg

Herzlichen Dank für die Unterstützung:

GOLD-Sponsor

IE Industrial Engineering München GmbH

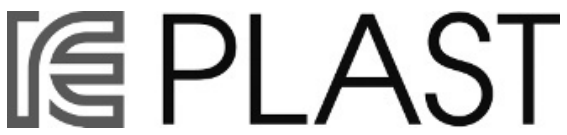

www.ie-group.com

BRONZE-Sponsor

LPKF WeldingQuipment GmbH 
https://doi.org/10.51202/9783182443469-

Generiert durch IP '172.22.53.54', am 26.04.2023, 15:00:42

Das Erstellen und Weitergeben von Kopien dieses PDFs ist nicht zulässig. 


\section{Inhalt}

Kunststoffprodukte lösen Anwenderprobleme

Zwischen Blut und Stahl - Einwegspritzgussteile im diagnostischen Workflow - Herausforderungen für die diagnostische Industrie und ihre Spritzgusspartner

Dr. T. Schwarz, Roche Diagnostics International AG, Rotkreuz, Schweiz

Kunststoffe in Unfallchirurgie und Orthopädie - Indikationsbereiche und Erfahrungen

Prof. Dr. med. K. Weise, em. o. Prof. für Unfallchirurgie,

ehem. Ärztl. Direktor Berufsgen. Unfallklinik, Universität Tübingen

Regulatorische Trends - Chance oder Hürde?

Dr.-Ing. S. Habibi-Naini, pheneo $\mathrm{GmbH}$, Bremen

Daten als Kernfaktor für eine innovative Medizintechnik

Prof. Dr. A. Rossmann, Herman Hollerith Lehr- und Forschungszentrum,

Fakultät Informatik, Hochschule Reutlingen, Böblingen

Fortschritte bei Materialien und im Materialmanagement

PEEK als Hochleistungskunststoff in der Medizintechnik

F. Claus, Evonik Industries AG, Marl

Systematische Entwicklung modellbasierter Prüfungen für Medizinprodukte nach Richtlinie VDI 5703

M. Kruse, M.Sc., Dipl.-Ing. V. N. Gesche, Prof. Dr. med. S. Jockenhövel, Abteilung Medizinische Fasersysteme, Bereich Medizinische Textilien, Institut für Textiltechnik der RWTH Aachen

Nanotechnologien in der Medizintechnik

Zulassung und Implementierung neuer Nanotechnologien in der Medizintechnik - VDI Richtlinie NanoMed / VDI 5710 Entwurf Dipl.-Ing W. Kexel, TÜV Technische Überwachung Hessen GmbH

Die neue ISO-Norm 10993-22 - „Guidance on Nanomaterials“

Dr. A. Poth, Eurofins BioPharma Product Testing, München 
Herstellung und Charakterisierung von Nanopartikeln für medizinische Anwendungen

Dr. S. Dembski, Fraunhofer ISC, Würzburg

Oberflächen: Wenige Nanometer moderieren die Wechselwirkung mit biologischen Systemen

C. Oehr, J. Barz, K. Borchers, M. Müller,

Fraunhofer-Institut für Grenzflächen- und Bioverfahrenstechnik, Stuttgart

Nanomaterialien in der Medizin: Es bleiben offene Fragen

Prof. Dr.-Ing. J. Vienken, Nephro-Solutions AG, Hamburg

3D-Druck und Spritzgießen als komplementäre Technologien

Fakten und Trends zur Additiven Fertigung in der Medizintechnik Prof. A. Gebhardt, J. Kessler, MEng,

GotheLab for Additive Manufacturing, Aachen

Biokompatible Materialien für 3D-gedruckte Medizinprodukte

Dr. H. Wiese, Dreve ProDiMed Unna

Time-to-Market bei Medizinprodukten: Herausforderungen und Potenziale des 3D-Drucks

Dr.-Ing. M. Schönberger, FRANK plastic AG, Waldachtal

Ergonomie \& Usability - Motoren der Produktentwicklung

Usability 3.0 - Patientensicherheit und Wettbewerbsvorteil

Dipl.-Ing T. Gruchmann, Use-Lab GmbH, Steinfurt

RFID Anwendungen in der Diagnostik und Medizintechnik

K. Eggmann, G. Kotyrba,

WEIDMANN MEDICAL TECHNOLOGYAG, Rapperswil, Schweiz

Optimierung des Fertigungsprozesses

Spritzgießwerkzeuge in der Medizintechnik: Nachweis richtlinienkonformer Inbetriebnahme und Produktionsabläufe

A. Müller (M.Eng.), Prof. Dr.-Ing. T. Seul, Hochschule Schmalkalden, Labor für Angewandte Kunststofftechnik, Schmalkalden 
Next generation vollautomatische und hochauflösende $100 \%$ Kontrolle von Stents

R. Eijck, GDO B.V., Eygelshoven, Niederlande

Laser-Kunststoffschweißen in der Medizintechnik -

Klar-Klar-Schweißen von mikrofluidischen Anwendungen mit dem LPKF

ClearJoining Verfahren

Dipl.-Ing. R. Bühring, LPKF WeldingQuipment GmbH, Fürth

\section{Neue Medizinprodukte in Forschung und Entwicklung}

Entwicklung einer patientenoptimierten Bandscheibentotalendoprothese Von einer Idee zum Implantatkonzept

Dipl.-Ing. E. Krampe, Polymer Consulting Krampe, Ismaning;

Dipl.-Ing. M. Haerst, Prof. Dr. med. Dr.-Ing. habil. E. Wintermantel,

Institute of Medical and Polymer Engineering,

Lehrstuhl für Medizintechnik, TU München, Garching

Funktionalisierte Mikroformschläuche - Projektilinjektionstechnik für

Flüssigsiliconkautschuk

Prof. Dr.-Ing. Ch. Hopmann, Dipl.-Ing. D. Kaltbeitzel,

Institut für Kunststoffverarbeitung an der RWTH Aachen, Aachen

Strömung und Thermodynamik im Reinraum: Wunschdenken versus Realität Prof. Dipl.-Ing. P. Karlinger, Hochschule Rosenheim;

Prof. Dipl.-Ing. G. Dittel, Dittel Engineering

Ein Hauch von Atem: Flüchtige Metaboliten in der Ausatemluft -

Sensorkopfkissen und Funktionstextilien

Prof. Dr. J. I. Baumbach, Fakultät Angewandte Chemie,

Hochschule Reutlingen

Umsetzung von Qualitätsanforderungen

Anforderungen an Medizinprodukte aus regulatorischer Sicht

M. Schäfer, Qualitätsmanagement und Produktzulassung in der Medizintechnik, Hechingen 
https://doi.org/10.51202/9783182443469-

Generiert durch IP '172.22.53.54', am 26.04.2023, 15:00:42

Das Erstellen und Weitergeben von Kopien dieses PDFs ist nicht zulässig. 